\title{
Antidepressant activity of $\omega-3$ polyunsaturated fatty acids in ovariectomized rats: role of neuroinflammation and microglial polarization
}

Bin Wu ${ }^{1 \dagger}$, Qingen Song ${ }^{2 \dagger}$, Yongkang Zhang ${ }^{2}$, Changshui Wang ${ }^{3}$, Mengqi Yang ${ }^{3}$, Jun Zhang ${ }^{4}$, Wenxiu Han $^{3}$ and Pei Jiang ${ }^{3^{*}}$

\begin{abstract}
Background: Menopause predisposes individuals to affective disorders, such as depression, which is tightly related to neuroinflammation. While the neuroinflammatory condition has been demonstrated in ovariectomized (OVX) rodents, there is limited evidence concerning microglial polarization, a key process in brain immune activation, in menopause-related brain.

Methods: Therefore, the present study aims to evaluate the polarized microglia in long-term OVX rats and we further explored whether supplementation of $\omega-3$ polyunsaturated fatty acids (PUFA), the pleiotropic bioactive nutrient, is effective in the neurobehavioral changes caused by OVX.

Results: Our data showed that OVX-induced anxiety and depression-like behaviors in rats, accompanied with increased neural apoptosis and microglial activation in the hippocampus. Additionally, OVX enhanced proinflammatory cytokines expression and suppressed the expression of anti-inflammatory cytokine, IL-10. Correspondingly, OVX reinforced NFKB signaling and shifted the microglia from immunoregulatory $\mathrm{M} 2$ phenotype to proinflammatory M1 phenotype. Meanwhile, daily supplementation with PUFA suppressed microglial M1 polarization and potentiated M2 polarization in OVX rats. In parallel, PUFA also exerted antidepressant and neuroprotective activities, accompanied with neuroimmune-modulating actions.
\end{abstract}

Conclusion: Collectively, the present study firstly demonstrated the disturbed microglial polarization in the OVX brain and provide novel evidence showing the association between the antidepressant actions of PUFA and the restraint neuroinflammatory progression.

Keywords: Depression, Ovariectomy, Polyunsaturated fatty acids, Microglial polarization

\footnotetext{
* Correspondence: jiangpeicsu@sina.com

${ }^{\dagger}$ Bin Wu and Qingen Song contributed equally to this work.

${ }^{3}$ Institute of Clinical Pharmacy \& Pharmacology, Jining First People's Hospital,

Jining Medical University, Jining 272000, China

Full list of author information is available at the end of the article
}

(c) The Author(s). 2020 Open Access This article is distributed under the terms of the Creative Commons Attribution 4.0 International License (http://creativecommons.org/licenses/by/4.0/), which permits unrestricted use, distribution, and reproduction in any medium, provided you give appropriate credit to the original author(s) and the source, provide a link to the Creative Commons license, and indicate if changes were made. The Creative Commons Public Domain Dedication waiver (http://creativecommons.org/publicdomain/zero/1.0/) applies to the data made available in this article, unless otherwise stated. 


\section{Background}

Menopause is strictly associated with affective disorders, whereas anxiety and depression are frequently-occuring and debilitating psychiatric diseases in menopause [1]. The occurrence menopausal disorders in both brain and periphery is related to the loss of ovarian function and estrogen deficiency. In this scenario, ovariectomized (OVX) rodents become a widely used animal model of menopause, which is generally referred to as surgical menopause [2]. Longterm after OVX, the animals develop a reliable predisposition to anxiety and depression-like behaviors [3]. Although OVX-induced hormonal deficiency is likely to be the cause of behavioral changes, the mechanisms underlying the brain pathological changes remain equivocal.

Neuroinflammation is recognized as a major contributor to depression. It has been reported that patients with depression are prone to have higher status of proinflammatory cytokines, including interleukin (IL)-1 $\beta$, IL-6 and tumor necrosis factor $\alpha$ (TNF $\alpha)$ in the periphery and central nervous system [4]. The stress-induced animal models of depression are also characterized with overproduction of proinflammatory mediators [5]. In support, treatment with the endotoxin, lipopolysaccharide (LPS), induces immune activation in both periphery and brain, resulting in depression-like behaviors [6]. Likewise, neuroinflammatory provocation has been repeatedly observed in OVX rodents, whereas antidepressant strategies, such as exercise, estrogen supplementation or inflammasome inhibition, also exerted immune-regulatory features $[3,7]$, strongly indicating a role for immune system in OVX-induced behavioral disturbance. Microglia is considered as the resident macrophage in the brain with a crucial role in neuroinflammatory progression. Like macrophage, microglia can polarize into proinflammatory M1 phenotype and immunoregulatory M2 phenotype, which is responsible for the production of proinflammatory or antiinflammatory cytokines, respectively [8].

$\omega-3$ polyunsaturated fatty acids (PUFA), the pleiotropic bioactive nutrient, contains anti-inflammatory and antidepressive activities [9]. Our previous researches demonstrated that PUFA can mitigate LPS-induced behavioral changes and restore overactivated neuroimmune function [10]. Although the neuroimmuneregulatory actions of PUFA has been found in various animal models, whether PUFA is effective in the immune activation induced by OVX remains unknown.

Therefore, the present study aims to evaluate phenotype of microlgia in the hippocampus of rats following longterm OVX and further to explore the immune-regulatory role of PUFA in the antidepressant mechanism.

\section{Materials and methods}

\section{Animals}

Female Sprague-Dawley rats about 12 -week old were housed under a temperature- controlled $\left(23 \pm 2{ }^{\circ} \mathrm{C}\right)$ and
12/12 h light/dark cycle environment, with free access to food and water. All animal studies were carried out in accordance with the Regulations of Experimental Animal Administration issued by the State Committee of Science and Technology of the People's Republic of China, with the approval of the Ethics Committee in Jining Medical University.

\section{PUFA supplementation and ovariectomy}

The rats were randomly divided into four groups $(n=6-$ 7): Sham-operated control group (Sham), PUFA, OVX and OVX + PUFA. Animals were bilaterally ovariectomized under anesthesia with sodium pentobarbital (50 $\mathrm{mg} / \mathrm{kg}$ ) through intraperitoneal injection. Following two small incisions, the ovaries, oviducts and top of the fallopian tubes were bilaterally clamped and removed in OVX group. After anesthesia, similar protocols were conducted in Sham group with the abdominal wall opened and the ovaries exteriorized but not removed to create similar stressful events. Refined fish oil was administrated daily by gavage $(1.5 \mathrm{~g} / \mathrm{kg})$ in PUFA and OVX + PUFA groups for PUFA treatment (approximately $340 \mathrm{mg} / \mathrm{g}$ for EPA, $240 \mathrm{mg} / \mathrm{g}$ for DHA, Sheng Tianyu Biotechnology, China) at the same day before OVX surgery. The treatment procedures lasted for 10 weeks before sacrifice. The dosage of PUFA was selected based on our preliminary research and previous experiment showing the robust antidepressant effect of PUFA $[10,11]$. The animals in Sham group were sacrificed at the diestrus phase to avoid the effects of estrus cycle. At the end of the 10 weeks, behavioral tests were performed and the rats were anesthetized with sodium pentobarbital $(50 \mathrm{mg} / \mathrm{kg})$. Blood was collected and the brain was quickly removed on the ice after cardiac perfusion with PBS. The left hemisphere of the brain was maintained in $4 \%$ paraformaldehyde and then embedded in paraffin, prepared for histopathological examination and immunofluorescent staining. For the right hippocampus, the tissue was dissected and homogenized, which was used for and Western blot and PCR analysis.

\section{Behavioral tests}

Elevated plus maze (EPM) test, a validated method to assess axiety-like behavior, was used in the present study [12]. In brief, the maze apparatus was a cross-shaped Plexiglas platform with two opposite open arms (OA, $50 \times 10 \mathrm{~cm}$ ) and two opposite closed arms (CA, $50 \times 10$ $\mathrm{cm})$, connected by a central platform $(\mathrm{CP}, 10 \times 10 \mathrm{~cm})$. The rats were placed at the center of the maze with the head facing one of the open arm. The observer should be at least $1 \mathrm{~m}$ away from the maze. Using the video camera mounted vertically above the maze to record the number of entries into the open and closed arms, and the time spent in each arm during the 5 min period. 
The paradigm of forced swim test (FST) is based upon the evaluation of immobility, as a measure of behavioral despair in stressful and inescapable situations [13]. In brief, each rat was placed in a glass cylinder $(45 \mathrm{~cm}$ height, $25 \mathrm{~cm}$ diameter) which contained approximately $35 \mathrm{~cm}$ of water $\left(24 \pm 1{ }^{\circ} \mathrm{C}\right)$ for $15 \mathrm{~min}$. The rats were then dried and transfered to their cage. They were placed again in same cylinders $24 \mathrm{~h}$ later for a $5 \mathrm{~min}$ swim test while it was videotaped. And the duration of immobility which defined as floating passively and only making slight movements to keep the head above water, was scored by an experienced observer blind to the experiment design.

Sucrose Preference Test (SPT) is diffusely used for the measurement of the depressive-like behavior in rats [14]. Prior to SPT, all the rats were housed individually. After two bottles of $1 \%$ sucrose solution placed on each cage for $48 \mathrm{~h}$, rats were deprived water for $14 \mathrm{~h}$. Then two pre-weighing bottles, one containing $1 \%$ sucrose solution and the other containing water, were placed to each rat. The side of the two bottles was randomly placed to reduce spatial bias and the bottles were weighed again after $1 \mathrm{~h}$. The weight difference was deemed to be the rat intake from each bottle. The preference for sucrose was measured as a percentage of the consumed $1 \%$ sucrose solution relative to the total amount of liquid intake.

The novelty-suppressed feeding test (NSFT) was adapted from previous study [15]. Before testing, rats were fasted for $24 \mathrm{~h}$. The rats were placed in an open field $(75 \times 75 \times 40 \mathrm{~cm})$. And a white paper $(10 \times 10 \mathrm{~cm})$ with a small amount of food was placed in the center of open field. Rats were allowed to move freely for $8 \mathrm{~min}$ in open field. Using a stopwatch, record the latency to feed, specifically the time it took for the rat to approach and take the first bite of the food. Immediately afterwards, the animals were transferred to their cage, and the total food intake belong the next $5 \mathrm{~min}$ was also weighed to avoid the influence of the appetite of rats.

\section{Hormone assay}

Serum were immediately separated by centrifuging at $4000 \mathrm{rpm}$ for $10 \mathrm{~min}$ at $4{ }^{\circ} \mathrm{C}$ and stored at $-80{ }^{\circ} \mathrm{C}$ until assay. The serum concentrations of $17 \beta$-estradiol were quantified using a commercial enzyme-linked immunosorbent assay (ELISA) kit (Guxi Biotech, China) according to the manufacturer's protocol.

\section{Apoptosis analysis}

The tissues were fixed in $10 \%$ phosphate-buffered paraformaldehyde for $48 \mathrm{~h}$ and then embedded in paraffin which prepared for immunohistochemical staining and histopathological examination. The presence of apoptosis was assessed by the terminal deoxynucleotidyl transferase-mediated FITC-dUTP nick end labeling (Tunel) method, which detects fragmentation of DNA in the nucleus during apoptotic cell death in situ. The apoptosis detection assay was performed using a commercially available kit following the manufacturer's protocol (Keygen Biotech, Nanjing, China). Three sections per mouse were used and the images of the hippocampal dentate gyrus (DG) region were analyzed.

\section{Immunostaining}

For the immunofluorescent analysis, paraffin-embedded cross sections of the tissues $(6 \mu \mathrm{m}$ thickness $)$ were dewaxed in xylol, rehydrated, antigen retrieval by boiling citric acid buffer (0.01 mol/L, pH 6.0) and rinsed in PBS. Then tissue sections were incubated with blocking $5 \%$ goat serum at $25^{\circ} \mathrm{C}$ for $1 \mathrm{~h}$. For immunocytochemistry, cells grown on coverslips were fixed with ice-cold $4 \%$ paraformaldehyde in PBS for $10 \mathrm{~min}$. Cells were blocked using $1 \% \mathrm{BSA}+0.3 \%(\mathrm{v} / \mathrm{v})$ Triton $\mathrm{X}-100+0.3 \mathrm{M}$ glycine (1\% BSA) in PBS at $25^{\circ} \mathrm{C}$ for $45 \mathrm{~min}$. The sections were then incubated with the primary antibody, anti-ionized calcium-binding adapter molecular 1 (Iba-1) (Abcam, 1: 200). The sections were washed with PBS three times and stained with DAPI (Beyotime Biotechnology, China) to stain the cell nuclei. Immunofluorescent images were taken with an inverted fluorescence microscope (Olympus, Japan) and analyzed by using the Image J software to obtain the mean fluorescence density of each visual field.

\section{Western blot analysis}

For western blotting analysis, total protein was prepared and the concentrations were analyzed using Bradford method. Samples were loaded on precast 12\% SDSPAGE gels with $50 \mu \mathrm{g}$ protein in each lane. Proteins in the gels were transferred to a PVDF membrane and blocked for $1 \mathrm{~h}$ in 5\% non-fat dry milk in TBS-T (25 mM Tris, $\mathrm{pH} 7.5,150 \mathrm{mM} \mathrm{NaCl}, 0.05 \%$ Tween-20). The following antibodies and concentrations were used over night at $4{ }^{\circ} \mathrm{C}$; phosphorylation of NF- $\mathrm{B}$ p 65 subunit (pp65, Proteintech, 1:500), p65 (Proteintech; 1:800), NF-кB inhibitor (IkB, Abcam, 1:1000), iNOS (Proteintech; 1: 500), Arginase-1 (Arg-1, Proteintech, 1:30000) and $\beta$ actin (Proteintech; 1:4000). After that, it was probed with HRP-conjugated secondary antibody for $40 \mathrm{~min}$. The signals were digitally scanned and was quantified using Image J software. The signals were normalized to $\beta$-actin as an internal standard.

\section{Real-time PCR analysis}

Total RNA was extracted by using Trizol reagent (invitrogen, USA) following the manufacturer's instructions. The RNA concentration and integrity was determined by spectrophotometry $(260-280 \mathrm{~nm}$ ) (Jingke, Ningbo, 
China). cDNA was generated from total RNA using the Revert Aid First Strand cDNA Synthesis Kit (Thermo Fisher Scientific, Tewksbury, MA, USA). Quantitative PCR was performed using gene-specific primers (Table 1) and SYBR green PCR kit (Applied Bio-systems, USA) on Bio-rad Cx96 Detection System (Bio-rad, USA). All PCR experiments was tested in triplicate. The PCR amplification program were: $50{ }^{\circ} \mathrm{C}$ for $2 \mathrm{~min}, 95^{\circ} \mathrm{C}$ for $10 \mathrm{~min}, 40$ cycles of amplification at $95^{\circ} \mathrm{C}$ for $15 \mathrm{~s}$ and $60{ }^{\circ} \mathrm{C}$ for 1 min. Relative quantitation for PCR product was normalized to $\beta$-actin as an internal standard.

\section{Statistical analysis}

All statistical procedures were performed on SPSS version 18. Data were expressed as mean \pm SD. Differences between groups were determined by two-way ANOVA. The prior level of significance was established at $p<0.05$. Tukey's test for post hoc comparisons were performed following any significant ANOVA.

\section{Results}

Effects of PUFA on body weight, uterine weight, estradiol and behaviors

As shown in Table 2, OVX markedly decreased circulating estradiol status (main effect of OVX, $\mathrm{F}=82.77, p<0.01$ ) and uterine weight (main effect of OVX, F $=73.52, p<$ 0.01 ), indicating that the animal model was successfully built. In line with previous findings [16], OVX pronouncedly increased the body weight growth (main effect of OVX, $\mathrm{F}=41.03, p<0.01$ ). However, PUFA treatment only slightly but non-significantly increased estradiol levels with no significant effect on body weight gain and uterine weight. Nonetheless, PUFA exerted robust anti-anxiety properties in EPM test of OVX rats and there was a significant interaction between PUFA and OVX on anxietylike behaviors in time spent in open arms $(\mathrm{F}=16.09, p<$ 0.01) (Fig. 1a) and open arm entries ( $\mathrm{F}=24.73, p<0.01)$ (Fig. 1b). In addition, although both PUFA and OVX didn't affect sucrose preference in SPT (Fig. 1d), PUFA alleviated OVX-induced depressive-like behaviors with decreased immobility time in FST (PUFA $\times$ OVX interactions, $\mathrm{F}=5.82, p<0.05$ ) (Fig. 1e) and latency time to feed in NSFT (PUFA $\times$ OVX interactions, $\mathrm{F}=12.41, p<0.01$ ) (Fig. 1f).

\section{Neuroprotective effects of PUFA in OVX rats}

As shown in Fig. 2., chronic OVX induced the abundance of Tunel-positive cells compared with Sham group (main effect of OVX, $\mathrm{F}=36.91, p<0.01$ ), indicating that the apoptotic cell rate in the hippocampus was significantly increased after OVX, whereas the rate of apoptotic cells in the OVX + PUFA group was markedly reduced compared with OVX group (PUFA $\times$ OVX interactions, $\mathrm{F}=27.84, p<0.01)$. Likewise, Iba-1 staining was used for the analysis of microglia activation [17] and two-way ANOVA revealed a significant interaction between PUFA and OVX $(\mathrm{F}=15.63, p<0.01)$. Post hoc comparisons demonstrated that PUFA treatment ameliorated OVX-induced microglia activation with a significant decrease of Iba- 1 abundance $(p<0.01)$, suggesting potential anti-inflammatory effects.

\section{Effects of PUFA and OVX on cytokine expression and NF- KB signaling}

To further confirm the immune-regulatory effect of PUFA, the expression of inflammatory cytokines (Fig. 3) and NF-kB (Fig. 4) signaling were assessed. We observed a remarkable increase of pro-inflammatory cytokine expression (IL-1 $\beta$, IL- 6 and TNF $\alpha)(p<0.01)$ but a marked decrease of anti-inflammatory IL-10 expression in the brain following the 10-week OVX (main effect of OVX, $\mathrm{F}=6.24, p<0.05)$. PUFA add-on treatment produced a reliable anti-inflammatory action by decreasing the expression of IL- $1 \beta$, IL- 6 and TNF $\alpha$ and increasing IL-10 and IL-4 expression in OVX animals. The transcription factor NF- $\mathrm{KB}$ serves as a dominated factor in coordinating the expression of a wide range of genes that control inflammatory responses [18]. We found that OVX

Table 1 Primer sequences used for the qPCR analysis

\begin{tabular}{|c|c|c|}
\hline Gene & Sense Primer $\left(5^{\prime}-3^{\prime}\right)$ & Antisense Primer (5'-3') \\
\hline $\mathrm{IL}-1 \beta$ & AGGTCGTCATCATCCCACGAG & GCTGTGGCAGCTACCTATGTCTTG \\
\hline IL-6 & CACAAGTCCGGAGAGGAGAC & ACAGTGCATCATCGCTGTTC \\
\hline TNFa & GAGAGATTGGCTGCTGGAAC & GAGAGATTGGCTGCTGGAAC \\
\hline $\mathrm{IL}-10$ & GTITACCTGGTAGAAGTGATGCC & ССACTGCCTTGCTITTATTCTC \\
\hline $\mid \mathrm{LL}-4$ & ACAGGAGAAGGGACGCCAT & GAAGCCCTACAGACGAGCTCA \\
\hline CD86 & TAGGGATAACCAGGCTCTAC & CGTGGGTGTCTIITGCTGTA \\
\hline iNOS & AGTGGCAACATCAGGTCGG & CGATGCACAACTGGGTGAAC \\
\hline CD206 & AGTTGGGTTCTCCTGTAGCCCAA & ACTACTACCTGAGCCCACACCTGCT \\
\hline Arg-1 & GGGAAAAGCCAATGAACAGC & CCAAATGACGCATAGGTCAGG \\
\hline$\beta$-Actin & CATCCTGCGTCTGGACCTGG & TAATGTCACGCACGATTTCC \\
\hline
\end{tabular}


Table 2 Effect of $\omega-3$ PUFAs supplementation on body weight changes, uterine weight and serum estradiol status in ovariectomized $(\mathrm{OVX})$ rats

\begin{tabular}{llll}
\hline Group & Body weight gain $(\mathrm{g})$ & Uterine weight $(\mathrm{mg})$ & Estradiol $(\mathrm{pg} / \mathrm{ml})$ \\
\hline Control & $227.49 \pm 12.22$ & $621.68 \pm 106.31$ & $106.06 \pm 9.97$ \\
PUFA & $231.19 \pm 19.61$ & $592.19 \pm 77.09$ & $117.77 \pm 12.03$ \\
OVX & $278.95 \pm 10.92^{* *}$ & $155.84 \pm 32.62^{* *}$ & $29.26 \pm 5.37^{* *}$ \\
OVX + PUFA & $283.34 \pm 12.66$ & $161.25 \pm 48.07$ & $35.04 \pm 7.52$ \\
\hline
\end{tabular}

Data are means \pm SD $(n=7-9) .{ }^{* *} p<0.01$ compared to control group

decreased IKB protein expression (main effect of OVX, $\mathrm{F}=5.93, p<0.05)$ and increased the protein status of $\mathrm{p}-$ p65 (main effect of OVX, F $=32.51, p<0.01$ ) and p65 (main effect of OVX, $\mathrm{F}=11.79, p<0.01$ ), whereas supplementation of PUFA suppressed the overactivated NF$\kappa \mathrm{B}$ signaling with a marked increase of ІкB expression and a decrease of p65 phosphorylation level.

\section{Effects of PUFA and OVX on microglial polarization}

To further explore the microglial phenotype, the expression of biomarkers of proinflammatory M1 phenotype (CD86 and iNOS) and immunoregulatory M2 phenotype (CD206 and Arg-1) was analyzed [19]. In line with the proinflammatory environment caused by OVX, longterm OVX shifted the microglia from M2 to M1 phenotype with increased expression of M1 mediators but decreased expression of M2 biomarkers, which was also alleviated by co-treatment of PUFA during OVX state (Fig. 5).

\section{Discussion}

Depression is more frequently observed in women, and menopause is a critical predisposing factor for the psychiatric disorders. Although estrogen replacement therapy is reported effective on the mental disorder progression, there is a great concern of the side-effects of chronic estrogen treatment, such as breast cancer and cardiovascular events [20, 21]. Therefore, it becomes urgent to explore the underlying mechanisms of menopause-related depression and seek an alternative treatment strategy for the mental condition.

Our data showed that chronic OVX induced anxietylike and depression-like behaviors. Given previous research showed the time-dependent effects of OVX on

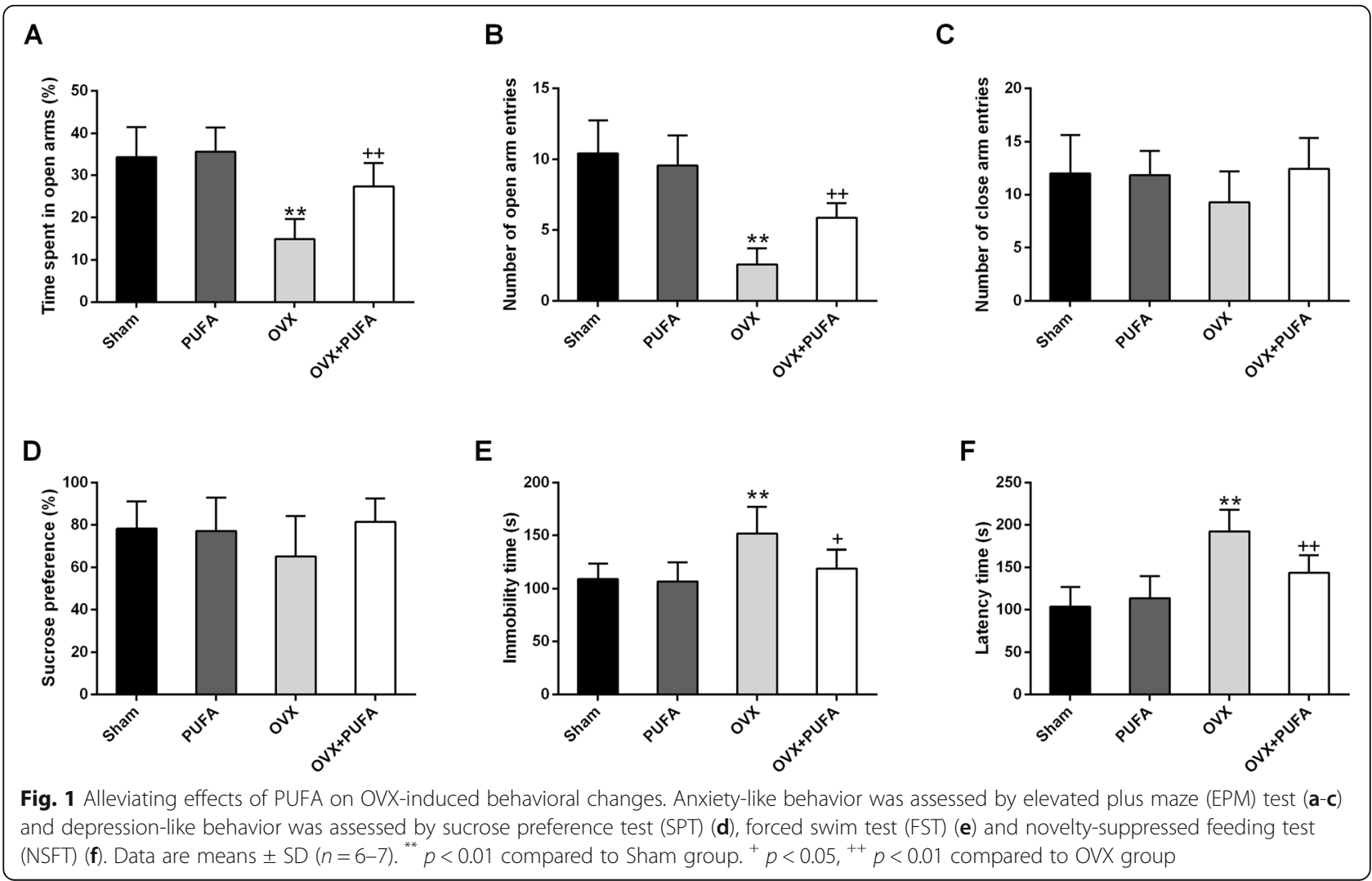




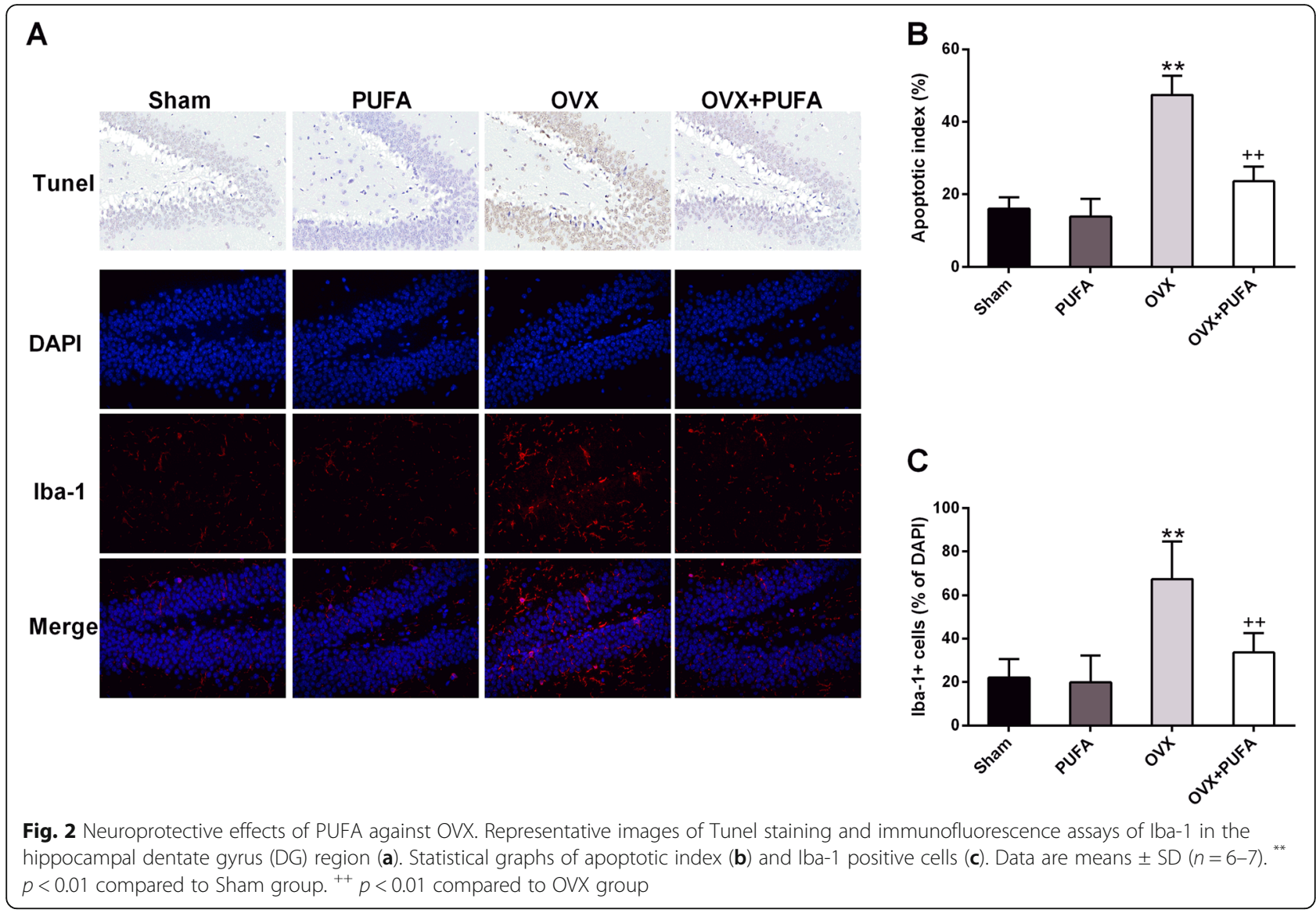

behavioral changes that at least 6 weeks are needed for the animal to develop depression-like behaviors [22], the present study evaluated the behaviors and biochemical factors 10 weeks after OVX surgery to guarantee the animal model was successfully developed and PUFA was sufficiently supplemented. The experimental procedure succeeded as evidenced by the increased body weight and decreased uterine weight and estrogen levels. It should be noted that these basic characteristics were not changed by PUFA, indicating that the antidepressant effects is estrogen-independent. In support, it has been found that PUFA contains robust antidepressant effects in both clinical trials and chronic stress-induced depression-like rodents [23, 24]. Additionally, we previously found that PUFA administration mitigates neuroinflammation and depression-like behaviors in animals exposed to repeated LPS challenge [10]. In fact, the antidepressant effect of PUFA is, at least partially, attributed to its immune-modulating actions and the inflammatory progression is tightly linked to the depression-line behaviors caused by either chronic stress, LPS or OVX.

As expected, PUFA also ameliorated the neuronal apoptosis and neuroimmune overactivation caused by OVX as evidenced by the fact that the Tunel-positive cells and Iba-1 immunofluorescent abundance were remarkably decreased in OVX + PUFA group in comparison with OVX group. As previously reported [25], long-term following OVX induced the animals to a neuroimmuneactivated condition with increased expression of proinflammatory cytokines but decreased expression antiinflamamtory cytokine. The neuroinflammatory state is indicated to affect every pathological aspect of depression, including neurogenesis, neuronal apoptosis, neurotransmission and neuroplasticity, playing a major role in the onset and development of the mental disorder [26]. Microglia is the predominant resident immune cells in the brain responding to stressful stimuli. Generally, microglia can be categorized into two types, the classic proinflammatory M1 type and immunoregulatory M2 phenotype, which is responsible for the production of proinflammatory or anti-inflammatory cytokines, respectively. Although the switch of the M1/M2 microglial phenotypes is recently regarded as a novel therapeutic strategy for depression [27], there is no research evaluating microglial polarization in the OVX brain. The present research firstly showed that chronic OVX shifted the microglial polarization from M2 toward M1 phenotype as evidenced by the increased M1 biomarker (CD86 and iNOS) but decreased M2 biomarker (CD206 and Arg-1), which corresponded with the altered expression of inflammatory 


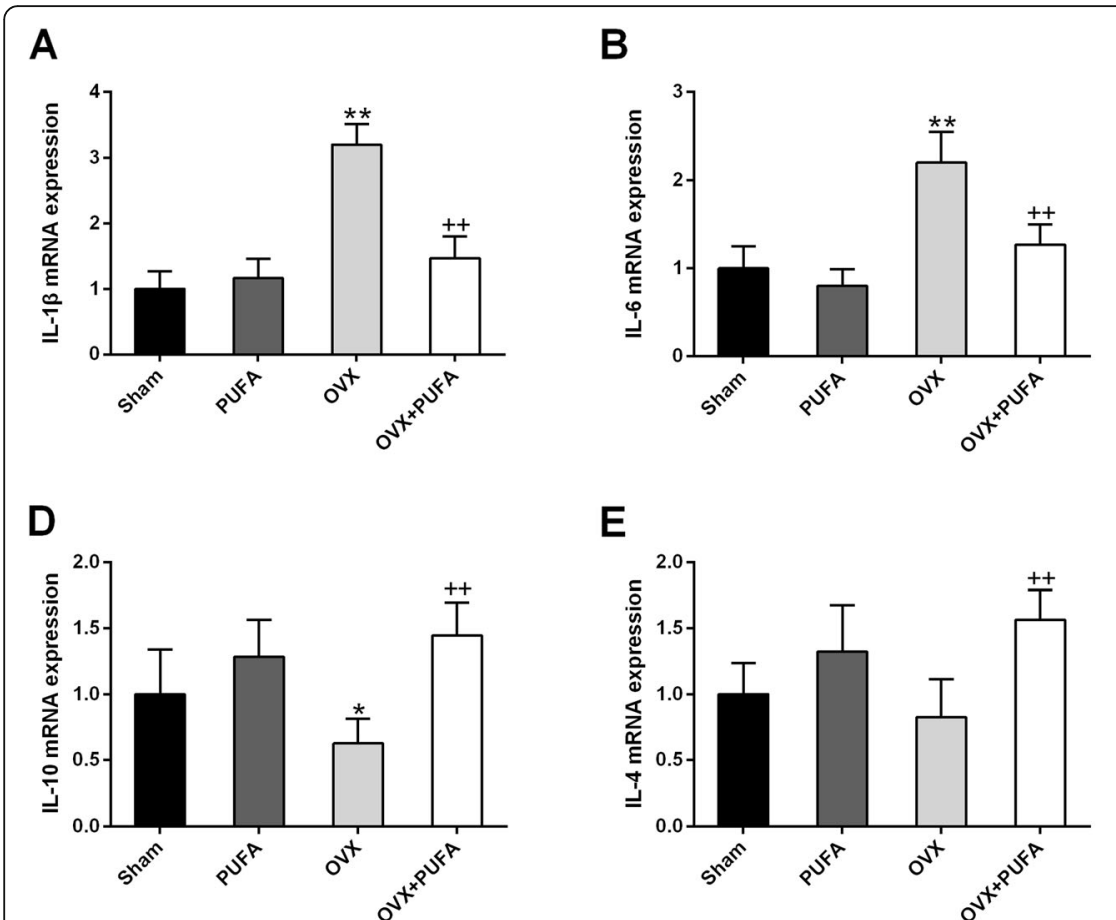

Fig. 3 Anti-inflammatory effects of PUFA in OVX rats. Relative mRNA expression of proinflammatory cytokines, IL-1 $\beta$ (a), IL-6 (b) and TNFa (c), and anti-inflammatory cytokines, IL-10 (d) and IL-4 (e). Data are means \pm SD $(n=6-7) .{ }^{*} p<0.05,{ }^{* *} p<0.01$ compared to Sham group. ${ }^{++} p<0.01$ compared to OVX group

A

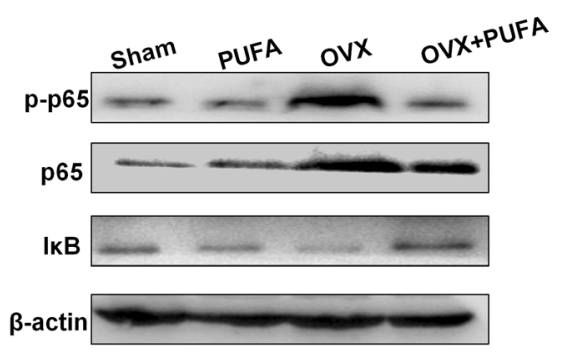

C

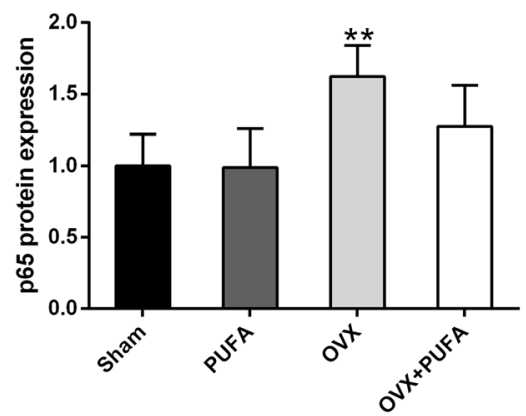

B

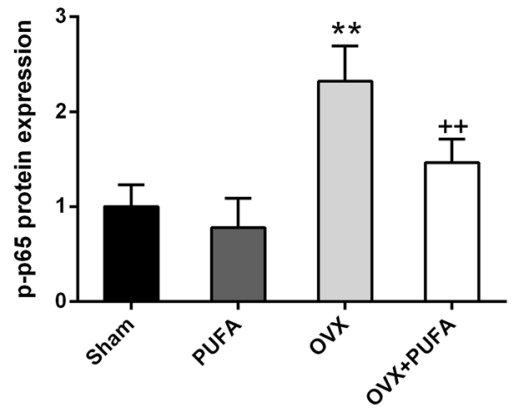

D

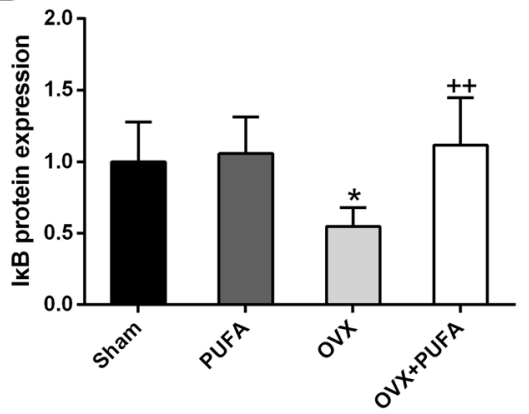

Fig. 4 Effects of PUFA and OVX on NF-KB signaling. Representative blots (a) and statistical graphs of p-p65 (b), p65 (c) and IkB (d) expression. Data are means $\pm \mathrm{SD}(n=6-7) .{ }^{*} p<0.05,{ }^{* *} p<0.01$ compared to Sham group. ${ }^{++} p<0.01$ compared to OVX group 


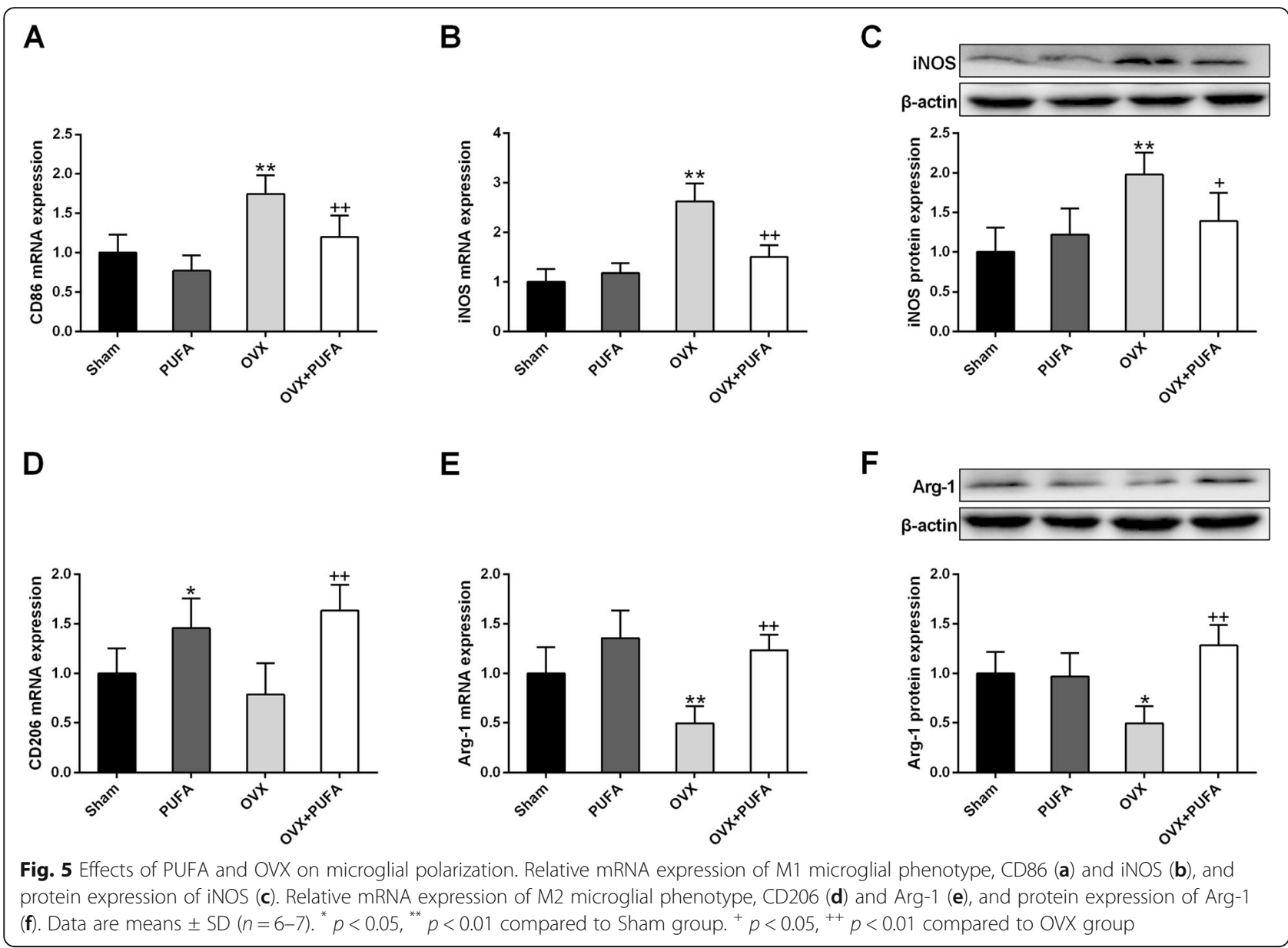

cytokines. Meanwhile, in accordance with the findings in the animal models of LPS or stress-induced depression [10], the anti-inflammatory and anti-depressant properties of PUFA were also verified in this OVX model. These beneficial effects are very likely to be associated with the phenotypic transition of microglia, as the present study showed that PUFA supplementation maintained the normal homeostatic balance between the M1 and the M2 phenotypes.

NF- $\mathrm{kB}$ serves as a dominated factor in coordinating the expression of a wide range of genes that control inflammatory responses and one of the proinflammatory mechanisms is that the activation of NF- $\mathrm{kB}$ signaling pathway is an essential trigger for the phenotypic transition from M2 to $\mathrm{M} 1$ phenotype [28]. Under normal physiological condition, NF- $\mathrm{KB}$ maintains in an inactive state in the cytoplasm, where it is bound to the inhibitor IкB. Once activated, IKB is phosphorylated and degraded, resulting in p65 phosphorylation and translocation, triggering inflammatory progression [29]. To provide the mechanistic insight, the key components of NF- $\mathrm{kB}$ pathway were assessed. The OVX procedures induced I $\mathrm{KB}$ degradation and enhanced the expression and phosphorylation of $\mathrm{p} 65$, indicating NF- $\mathrm{kB}$ signaling was activated, thereby shifting the microglial polarization from M2 toward M1 phenotype and promoting the transcription of proinflammatory cytokines. Intriguingly, PUFA co-treatment effectively rebalanced NF- $\kappa B$ signaling, preventing the pathway from overactivation induced by OVX. These results highlighted the involvement of NF- $\mathrm{kB}$ pathway underlying the antiinflammatory and microglial regulatory mechanisms of PUFA in the condition of OVX.

\section{Conclusions}

Collectively, the present study firstly showing the alterations of microglial polarization in OVX rats, shedding insight into the mechanisms of neuroinflammation and altered mood in menopause phase. Additionally, we found that PUFA also contains neuroimmune-regulatory and antidepressant effects in the context of OVX. These findings are interesting given accumulating evidence shows the protective actions of PUFA against cardiovascular hepatic dysfunction caused by OVX, providing novel evidence for the supplementary need of the multifunctional nutrient, PUFA, in menopause. 


\section{Abbreviations}

CA: Closed arms; CP: Central platform; FSTforced swim test; ELISA: Enzymelinked immunosorbent assay; EPM: Elevated plus maze; IL-1 $\beta$ : Interleukin -1 $\beta$; IL-6: Interleukin - 6; LPS: Lipopolysaccharide; NSFT: Novelty-suppressed feeding test; OA: Open arms; OVX: Ovariectomized; PUFA: $\omega-3$ polyunsaturated fatty acids; SPT: Sucrose preference test; TNFa: Tumor necrosis factor a

\section{Acknowledgements}

Not applicable.

\section{Authors' contributions}

BW, QS, YZ, CW, MY, JZ, WH and PJ conceived the study, interpreted the data and andcritically revised the reports. BW, QS, YZ and CW contributed to the data acquisition. PJ participated in writing and revising the manuscript. All authors read and approved the final manuscript.

\section{Funding}

The study was supported by the National Natural Science Foundation of China (81602846) and Taishan Scholar Project of Shandong Province (tsqn201812159).

\section{Availability of data and materials}

The datasets used and analyzed during the current study are available fromthe corresponding author on reasonable request.

\section{Ethics approval and consent to participate}

All animal use procedures were carried out in accordance with the Regulations of Experimental Animal Administration issued by the State Committee of Science and Technology of the People's Republic of China, with the approval of the Ethics Committee in our university.

\section{Consent for publication}

All authors read and approve the final manuscript.

\section{Competing interests}

The authors declare that they have no competing interests.

\section{Author details}

'Department of Gynecology, Taian City Central Hospital, Taian, China. ${ }^{2}$ Jinxiang People's Hospital, Jining Medical University, Jining, China. ${ }^{3}$ Institute of Clinical Pharmacy \& Pharmacology, Jining First People's Hospital, Jining Medical University, Jining 272000, China. ${ }^{4}$ Department of Pharmacy, The First Affiliated Hospital of Zhengzhou University, Zhengzhou, China.

Received: 25 July 2019 Accepted: 5 January 2020

Published online: 08 January 2020

\section{References}

1. Georgakis MK, Thomopoulos TP, Diamantaras AA, Kalogirou El, Skalkidou A, Daskalopoulou SS, et al. Association of age at menopause and duration of reproductive period with depression after menopause: a systematic review and meta-analysis. JAMA psychiatry. 2016;73(2):139-49.

2. Zhang Z, Chen Y, Xiang L, Wang Z, Xiao GG, Hu J. Effect of Curcumin on the Diversity of Gut Microbiota in Ovariectomized Rats. Nutrients. 2017:9(10): 1146

3. Xu Y, Sheng H, Bao Q, Wang Y, Lu J, Ni X. NLRP3 inflammasome activation mediates estrogen deficiency-induced depression- and anxiety-like behavior and hippocampal inflammation in mice. Brain Behav Immun. 2016;56:175-86.

4. Jeon SW, Kim YK. Inflammation-induced depression: its pathophysiology and therapeutic implications. J Neuroimmunol. 2017;313:92-8.

5. Zhang WY, Guo YJ, Han WX, Yang MQ, Wen LP, Wang KY, et al. Curcumin relieves depressive-like behaviors via inhibition of the NLRP3 inflammasome and kynurenine pathway in rats suffering from chronic unpredictable mild stress. Int Immunopharmacol. 2019;67:138-44.

6. Gong X, Hu H, Qiao Y, Xu P, Yang M, Dang R, et al. The involvement of renin-angiotensin system in lipopolysaccharide-induced behavioral changes, Neuroinflammation, and disturbed insulin signaling. Front Pharmacol. 2019;10:318.
7. Wang Y, Xu Y, Sheng H, Ni X, Lu J. Exercise amelioration of depression-like behavior in OVX mice is associated with suppression of NLRP3 inflammasome activation in hippocampus. Behav Brain Res. 2016;307:18-24.

8. Ma Y, Wang J, Wang Y, Yang GY. The biphasic function of microglia in ischemic stroke. Prog Neurobiol. 2017;157:247-72.

9. Larrieu T, Laye S. Food for mood: relevance of nutritional Omega-3 fatty acids for depression and anxiety. Front Physiol. 2018;9:1047.

10. Dang R, Zhou X, Tang M, Xu P, Gong X, Liu Y, et al. Fish oil supplementation attenuates neuroinflammation and alleviates depressivelike behavior in rats submitted to repeated lipopolysaccharide. Eur J Nutr. 2018:57(3):893-906.

11. Wu YQ, Dang RL, Tang MM, Cai HL, Li HD, Liao DH, et al. Long chain Omega-3 polyunsaturated fatty acid supplementation alleviates doxorubicin-induced depressive-like behaviors and neurotoxicity in rats: involvement of oxidative stress and Neuroinflammation. Nutrients. 2016;8(4):243.

12. Liao D, Xiang D, Dang R, Xu P, Wang J, Han W, et al. Neuroprotective effects of dl-3-n-Butylphthalide against doxorubicin-induced Neuroinflammation, oxidative stress, endoplasmic reticulum stress, and behavioral changes. Oxidative Med Cell Longev. 2018;2018:9125601.

13. Yang M, Dang R, Xu P, Guo Y, Han W, Liao D, et al. Dl-3-n-Butylphthalide improves lipopolysaccharide-induced depressive-like behavior in rats: involvement of Nrf2 and NF-kappaB pathways. Psychopharmacology. 2018; 235(9):2573-85.

14. Jiang P, Guo Y, Dang R, Yang M, Liao D, Li H, et al. Salvianolic acid B protects against lipopolysaccharide-induced behavioral deficits and neuroinflammatory response: involvement of autophagy and NLRP3 inflammasome. J Neuroinflammation. 2017;14(1):239.

15. Liao D, Guo Y, Xiang D, Dang $R, X u P, C a i H$, et al. Dysregulation of Neuregulin-1/ErbB signaling in the hippocampus of rats after administration of doxorubicin. Drug design, development and therapy. 2018;12:231-9.

16. Iwasa T, Matsuzaki T, Yano K, Irahara M. The effects of ovariectomy and lifelong high-fat diet consumption on body weight, appetite, and lifespan in female rats. Horm Behav. 2018;97:25-30.

17. Tischer J, Krueger M, Mueller W, Staszewski O, Prinz M, Streit WJ, et al. Inhomogeneous distribution of Iba-1 characterizes microglial pathology in Alzheimer's disease. Glia. 2016:64(9):1562-72.

18. Lim H, Lee H, Noh K, Lee SJ. IKK/NF-kappaB-dependent satellite glia activation induces spinal cord microglia activation and neuropathic pain after nerve injury. Pain. 2017;158(9):1666-77.

19. Yang $X, X u S$, Qian $Y$, Xiao Q. Resveratrol regulates microglia M1/M2 polarization via PGC-1alpha in conditions of neuroinflammatory injury. Brain Behav Immun. 2017:64:162-72.

20. Anagnostis P, Paschou SA, Katsiki N, Krikidis D, Lambrinoudaki I, Goulis DG. Menopausal hormone therapy and cardiovascular risk. Where are we now? Curr Vasc Pharmacol. 2019:17(6):564-572.

21. Rondanina G, Puntoni M, Guerrieri-Gonzaga A, Marra D, Bonanni B, DeCensi A. Worry and risk perception of breast cancer in a prevention trial of low dose tamoxifen in midlife postmenopausal hormone users. Breast. 2017;34:108-14.

22. Puga-Olguin A, Rodriguez-Landa JF, Rovirosa-Hernandez MJ, GermanPonciano LJ, Caba M, Meza E, et al. Long-term ovariectomy increases anxiety- and despair-like behaviors associated with lower Fos immunoreactivity in the lateral septal nucleus in rats. Behav Brain Res. 2019; 360:185-95.

23. Deacon G, Kettle C, Hayes D, Dennis C, Tucci J. Omega 3 polyunsaturated fatty acids and the treatment of depression. Crit Rev Food Sci Nutr. 2017; 57(1):212-23.

24. Mathieu G, Denis $S$, Lavialle $M$, Vancassel $S$. Synergistic effects of stress and omega-3 fatty acid deprivation on emotional response and brain lipid composition in adult rats. Prostaglandins Leukot Essent Fat Acids. 2008; 78(6):391-401.

25. Park HJ, Shim HS, Chung SY, Lee TH, Shim I. Soyo-san reduces depressivelike behavior and proinflammatory cytokines in ovariectomized female rats. BMC Complement Altern Med. 2014;14:34.

26. Franklin TC, Xu C, Duman RS. Depression and sterile inflammation: essential role of danger associated molecular patterns. Brain Behav Immun. 2018;72:2-13.

27. Tang J, Yu W, Chen S, Gao Z, Xiao B. Microglia polarization and endoplasmic reticulum stress in chronic social defeat stress induced depression mouse. Neurochem Res. 2018;43(5):985-94.

28. Chen J, Sun Z, Jin M, Tu Y, Wang S, Yang X, et al. Inhibition of AGEs/RAGE/ rho/ROCK pathway suppresses non-specific neuroinflammation by 
regulating BV2 microglial M1/M2 polarization through the NF-kappaB pathway. J Neuroimmunol. 2017;305:108-14.

29. Seo EJ, Fischer N, Efferth T. Phytochemicals as inhibitors of NF-kappaB for treatment of Alzheimer's disease. Pharmacol Res. 2018;129:262-73.

\section{Publisher's Note}

Springer Nature remains neutral with regard to jurisdictional claims in published maps and institutional affiliations.

Ready to submit your research? Choose BMC and benefit from:

- fast, convenient online submission

- thorough peer review by experienced researchers in your field

- rapid publication on acceptance

- support for research data, including large and complex data types

- gold Open Access which fosters wider collaboration and increased citations

- maximum visibility for your research: over $100 \mathrm{M}$ website views per year

At $B M C$, research is always in progress.

Learn more biomedcentral.com/submissions 